\title{
ON THE REPRESENTATION OF THE SOLUTION OF A CLASS OF STOCHASTIC DIFFERENTIAL EQUATIONS
}

\section{RICHARD BELLMAN}

1. Introduction. In previous papers, $[1 ; 2]$, we have discussed the representation of the solutions of various classes of nonlinear differential equations in terms of the maximum operation. In this paper, we wish to indicate the application of similar techniques to the representation of the distribution function of the solution of stochastic differential equations. In the case where the equation has the form

$$
d u / d t=g(u)+r(t), \quad u(0)=c,
$$

with $r(t)$ a random function with given distribution, of which a particularly important case is the Riccati equation

$$
d u / d t=u^{2}+r(t), \quad u(0)=c,
$$

the answer assumes an especially simple form.

The nonlinear equation in (1.2) is associated with the linear equation

$$
d^{2} w / d t^{2}-r(t) w=0
$$

which plays a role in the study of wave propagation through a random medium.

2. Quasi-linearization. Let us make the fundamental assumption that $g(u)$ is a strictly convex twice differentiable function of $u$ for $-\infty<u<\infty$. Then we may write

$$
g(u)=\operatorname{Max}_{v}\left(g(v)+(u-v) g^{\prime}(v)\right) .
$$

The equation in (1.1) can then be written in the form

$$
\frac{d u}{d t}=\underset{v}{\operatorname{Max}}\left[g(v)+(u-v) g^{\prime}(v)\right]+r(t), \quad u(0)=c .
$$

It follows that we have the inequality

$$
\frac{d u}{d t} \geqq g(v)+(u-v) g^{\prime}(v)+r(t), \quad u(0)=c,
$$

valid for any function $v(t)$. Let $U(v ; t)$ denote the solution of the equation

Received by the editors August 3, 1957. 


$$
\frac{d U}{d t}=g(v)+(U-v) g^{\prime}(v)+r(t), \quad U(0)=c .
$$

Then, as in $[1 ; 2]$, we have the inequality

$$
u \geqq U(v ; t),
$$$$
t \geqq 0,
$$

for all $v$.

3. Representation of the probability distribution for $U$. From the relation given in (2.4) we derive the obvious inequality

$$
\operatorname{Prob}(u \geqq x) \geqq \operatorname{Prob}(U(v ; t) \geqq x)
$$

for $-\infty<x<\infty$ and all random functions $v(t)$.

Since we have equality for $v=u$, we can state the following result.

THEOREM. Let $u(t)$ be the random function determined by the solution of the stochastic differential equation in (1.1), assumed to exist for $0 \leqq t \leqq T$, and assume that $g(u)$ is a strictly convex twice differentiable function of $u$ for $-\infty<u<\infty$.

Then, for $0 \leqq t \leqq T$ and $-\infty<x<\infty$, we have the representation

$$
\operatorname{Prob}(u \geqq x)=\underset{\bullet}{\operatorname{Max}} \operatorname{Prob}(U(v ; t) \geqq x),
$$

where the maximization is over all random functions $v(t)$ defined over $0 \leqq t \leqq T$.

4. Discussion. It is easy to see that corresponding results can be obtained for nonlinear stochastic functional equations of the form

$$
L(u)=N(u)+r(t),
$$

whenever $N(u)$ has the required convexity, or concavity, property and $L(u)$ possesses the requisite positivity property, following the general pattern of the discussion in $[1 ; 2]$.

\section{REFERENCES}

1. R. Bellman, Functional equations in the theory of dynamic programming-II: Nonlinear differential equations, Proc. Nat. Acad. Sci. vol. 41 (1955) pp. 482-486.

2. - Functional equations in the theory of dynamic programming-V: Positivity and quasi-linearity, Proc. Nat. Acad. Sci. vol. 41 (1955) pp. 743-746.

RAND CORPORATION 